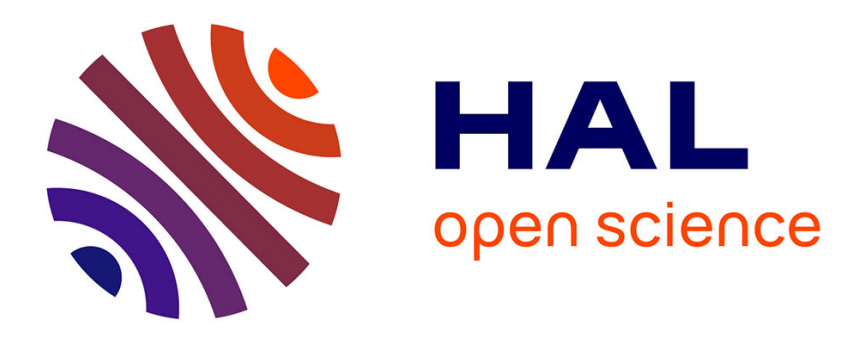

\title{
Piecewise affine output feedback controller for vehicle lane keeping
}

Andre Benine-Neto, Saïd Mammar

\section{To cite this version:}

Andre Benine-Neto, Saïd Mammar. Piecewise affine output feedback controller for vehicle lane keeping. American Control Conference (ACC 2012), Jun 2012, Montreal, Canada. pp.6533-6538, 10.1109/ACC.2012.6314735 . hal-00866996

\section{HAL Id: hal-00866996 https://hal.science/hal-00866996}

Submitted on 5 Jul 2021

HAL is a multi-disciplinary open access archive for the deposit and dissemination of scientific research documents, whether they are published or not. The documents may come from teaching and research institutions in France or abroad, or from public or private research centers.
L'archive ouverte pluridisciplinaire HAL, est destinée au dépôt et à la diffusion de documents scientifiques de niveau recherche, publiés ou non, émanant des établissements d'enseignement et de recherche français ou étrangers, des laboratoires publics ou privés. 


\title{
Piecewise Affine Output Feedback Controller for Vehicle Lane Keeping
}

\author{
André Benine-Neto ${ }^{1}$, Saïd Mammar ${ }^{2}$
}

\begin{abstract}
This paper presents the design and simulation test of a piecewise affine output-feedback controller for vehicle lane keeping. The design of the proposed lane keeping system takes into account the entire domain of lateral tire forces through piecewise affine approximations of the tire forces nonlinear behavior. The computation of the control law is casted as Bilinear Matrix Inequalities optimization procedure which is solved using the V-K-method to find simultaneously a piecewise quadratic Lyapunov function and the piecewise affine regulator and estimator structures. Simulation tests show the controlled car is able to well achieve the standard ISO-3888-2 transient maneuver.
\end{abstract}

\section{INTRODUCTION}

The development of autonomous vehicles has increased attention of researchers in the recent years. An overview of these activities is presented in [16]. Regarding vehicle lateral control, it can be noted that the research has evolved from automated lane keeping in which the lateral accelerations are generally low (see [15] and [9]) to more recent work which deals with the control of the vehicle in extreme dynamics to avoid accidents ([8] and [2]). For that, it is required to develop systems that are able to operate in the complete domain of tire forces, including the nonlinear behavior, as many accidents occurs due to the vehicle loss of control when the tire forces are saturated.

The lane keeping assistance system (LKAS) presented in [8] takes into account the nonlinear behavior of the lateral tire forces for the design of a parallel distributed controller based on a Takagi-Sugeno model. In [2] a Piecewise Affine (PWA) state feedback controller is developed to avoid the tire saturation on the lane departure avoidance maneuvers.

PWA systems have also been used in the development of other driver assistance systems. In [5] the longitudinal tire forces are approximated by PWA functions for a traction control system. The lateral tire forces have also been considered in PWA form to design yaw rate controllers in [4], [3] and [12].

In this paper, similar approximations of the lateral tire forces are used to design an output-feedback PWA controller for a LKAS, which is able to operate in the entire domain of the tire forces. As an extension of the work presented in [2], the measurement of the vehicle sideslip angle requires sensors that are not available in currently commercialized vehicles, therefore an estimator is designed to drive the switches of the PWA controller. Following the results obtained in [14], the control synthesis is casted as

\footnotetext{
${ }^{1}$ IFSTTAR - LIVIC, 14 Route de la Minière, 78000 Versailles, France (andre.benine-neto@ifsttar.fr)

3 IBISC - EA 4526 UEVE, 40 rue du Pelvoux CE1455, 91020, Evry, Cedex, France (e-mail: said.mammar@inrets.fr)
}

a Bilinear Matrix Inequality (BMI) optimization problem which is solved using the $\mathrm{V}-\mathrm{K}$ method. This interactive procedure allows the BMI to be transformed into two Linear Matrix Inequalities (LMI) optimization problems which can be solved more efficiently to find simultaneously the output feedback gain and a Piecewise Quadratic Lyapunov (PWQL) function that ensures stability of the closed loop system. The vehicle model for control synthesis is described in Section II, followed by the controller design shown in Section III. The simulation results obtained on a nonlinear model are presented in Section IV. Section V wraps up the work and provides some perspective for the future work.

\section{VEHICLE MODEL}

In order to design the control law for the LKAS, a PWA system is derived from a simple nonlinear vehicle model, in which the lateral translational and yaw motions are considered and the roll and pitch motions are neglected. The wheels of the front and rear axles are lumped into one located at the axle center leading to a bicycle model. The equations describing this model are given by:

$$
\left\{\begin{aligned}
m v(\dot{\beta}+r) & =f_{s f}+f_{s r} \\
J \dot{r} & =l_{f} f_{s f}-l_{r} f_{s r},
\end{aligned}\right.
$$

where the involved variables consist of the vehicle yaw rate, denoted $r$ and the vehicle sideslip angle, $\beta$. Concerning the fixed parameters for the model, $v$ represents the longitudinal vehicle speed, $m$ is the vehicle mass, $l_{f}\left(l_{r}\right)$ is the distance from the front (rear) axle to the center of gravity $(\mathrm{CG}), J$ is the vehicle inertia with respect to the vertical axle through the CG. All numerical values of these parameters are presented in Table I. The lateral forces $f_{s x}$, with $x=f, r$, for the front and rear tires can be modeled according to the Pacejka tire model [11]:

$f_{s x}\left(\alpha_{x}\right)=D_{x} \sin \left(C_{x} \operatorname{atan}\left(\left(1-E_{x}\right) B_{x} \alpha_{x}+E_{x} \operatorname{atan}\left(B_{x} \alpha_{x}\right)\right)\right)$,

where $\alpha_{x}$ stands for the front (rear) tire sideslip angle. Considering that the angles remain small, the sideslip angles for front and rear tires are given by:

$$
\alpha_{f}=\delta_{f}-\beta-\frac{l_{f} r}{v}, \quad \alpha_{r}=-\beta+\frac{l_{r} r}{v},
$$

where $\delta_{f}$ is the steering angle.

The Pacjeka tire model is depicted by the solid line in Fig. 1.

The simple nonlinear model (1) shows, as well known in literature (see for example [10]), a limited stability region which also depends on the driver steering wheel angle, two unstable equilibrium points and a stable one (bifurcation 


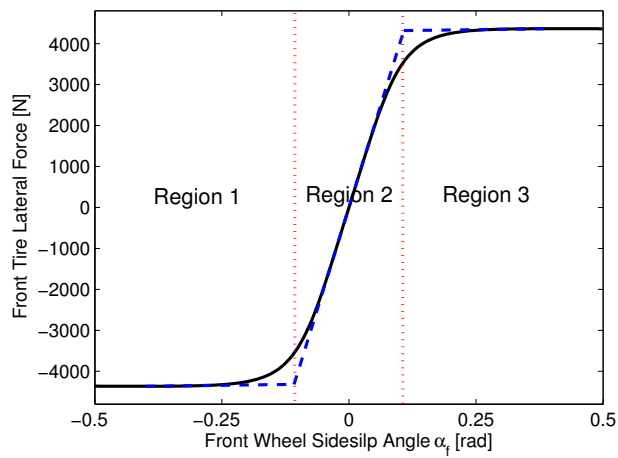

Fig. 1. Front tire forces described by the Pacejka magic formula and corresponding PWA approximations and partitions.

analysis). The causes of the instability are due to the nonlinear behavior of the tire-road forces which are taken into account in the PWA vehicle model described below.

\section{A. Piecewise affine approximation of lateral tire forces}

Considering that the front and rear tire forces described by (2) are approximated by the following PWA functions:

$$
\left\{\begin{array}{c}
f_{s f}\left(\alpha_{f}\right)=e_{f i}+d_{f i} \alpha_{f} \\
f_{s r}\left(\alpha_{r}\right)=e_{r i}+d_{r i} \alpha_{r}
\end{array}\right.
$$

where $d_{f i}, d_{r i}, e_{f i}$ and $e_{r i}$ depend on the front and rear tire forces and the index $(i)$ corresponding to the partitioning of the tire force domain, based on the tire sideslip angles, as illustrated by the dotted lines on Fig. 1 for the front wheel. For the region containing the origin $(i=2)$, a simple linear approximation is considered, in this case $d_{f 2}=c_{f}, d_{r 2}=$ $c_{r}, e_{f 2}=0$, and $e_{r 2}=0$. The cornering coefficients are related to the Pacejka parameters as; $c f=B_{f} C_{f} D_{f}$, and $c_{r}=B_{r} C_{r} D_{r}$

Replacing the PWA approximation of lateral tire forces (4) in the vehicle model (1) leads to the PWA system:

$$
\dot{x}^{d}=A_{i}^{d} x^{d}+B_{i}^{d} u+a_{i}^{d}
$$

with the corresponding dynamics:

$$
\begin{gathered}
A_{i}^{d}=\left[\begin{array}{cc}
-\frac{d_{f i}+d_{r i}}{m v} & -1-\frac{d_{f i} l_{f}-d_{r i} l_{r}}{m v^{2}} \\
\frac{d_{r i} l_{r}-d_{f i} l_{f}}{J} & -\frac{d_{f i} l_{f}^{2}+d_{r i} l_{r}^{2}}{J v}
\end{array}\right], \\
B_{i}^{d}=\left[\begin{array}{c}
\frac{d_{f i}}{m \varphi_{j}} \\
\frac{d_{f i} l_{f}}{J}
\end{array}\right], \quad a_{i}^{d}=\left[\begin{array}{c}
\frac{e_{f i}+e_{r i}}{m v} \\
\frac{e_{f i} l_{f}-e_{r i} l_{r}}{J}
\end{array}\right] .
\end{gathered}
$$

where the control input is the front wheel steering angle $u=\delta_{f}$ and the state variables are the vehicle sideslip angle, $\beta$ and the vehicle yaw rate, $r, x^{d}=[\beta, r]^{T}$.

Even though this approximation can be refined, it is shown in the simulation section that improved performance can be obtained by simply approximating the tire forces with the proposed PWA functions.
TABLE I

VALUES OF THE VEHICLE PARAMETERS.

\begin{tabular}{|l|l|}
\hline Parameter & Value \\
\hline$c_{f}$, front cornering stiffness & $40000 \mathrm{~N} / \mathrm{rad}$ \\
$c_{r}$, rear cornering stiffness & $35000 \mathrm{~N} / \mathrm{rad}$ \\
$l_{f}$, distance form CG to front axle & $1.22 \mathrm{~m}$ \\
$l_{r}$, distance form CG to rear axle & $1.44 \mathrm{~m}$ \\
$l_{s}$, look-ahead distance & $0.95 \mathrm{~m}$ \\
$\mathrm{~m}$, total mass & $1600 \mathrm{~kg}$ \\
$J$, vehicle yaw moment of inertia & $2454 \mathrm{kgm}$ \\
$v$, longitudinal velocity & $17 \mathrm{~m} / \mathrm{s}$ \\
\hline
\end{tabular}

\section{B. Additional dynamics for lane keeping}

For lane keeping purposes, the model (5) has to be expanded with the dynamics of the relative yaw angle and the lateral displacement with respect to the lane centerline. These measurements are provided by a video sensor. Let $\psi_{L}=$ $\psi-\psi_{d}$ be the yaw angle error which is the angle between the vehicle orientation and the tangent to the road. The road reference curvature $\rho_{\text {ref }}$ is defined by $\left(\dot{\psi}_{d}=v \rho_{\text {ref }}\right)$, and the following equality can be derived:

$$
\dot{\psi}_{L}=r-v \rho_{\text {ref }} .
$$

Denoting by $l_{s}$ the look-ahead distance, the equation giving the evolution of the measurement of the lateral offset $y_{L}$ from the centerline at sensor location is obtained by

$$
\dot{y}_{L}=v\left(\beta+\psi_{L}\right)-l_{s} r .
$$

Equations (8) and (9) are included in system (5) and the PWA system describing the vehicle dynamics and positioning is described by:

$$
\dot{x}^{p}=A_{i}^{p} x^{p}+B_{i}^{p} u+B_{\rho}^{p} \rho_{\text {ref }}+a_{i}^{p}
$$

where $x^{p}=\left[\beta, r, \psi_{L}, y_{L}\right]^{T}$ and

$$
\begin{aligned}
A_{i}^{p} & =\left[\begin{array}{cccc}
A_{i}^{d} & 0 & 0 \\
0 & 1 & 0 & 0 \\
v & l_{s} & v & 0
\end{array}\right], \\
B_{i}^{p} & =\left[\begin{array}{lll}
\left(B_{i}^{d}\right)^{T} & 0 & 0
\end{array}\right]^{T}, \\
B_{\rho}^{p} & =\left[\begin{array}{llll}
0 & 0 & -v & 0
\end{array}\right]^{T}, \\
a_{i}^{p} & =\left[\begin{array}{lll}
\left(a_{i}^{d}\right)^{T} & 0 & 0
\end{array}\right]^{T} .
\end{aligned}
$$

An illustration of the state variables is provided in Fig. 2

\section{Control Strategy}

The algorithm presented in [14] is applied for the design of the proposed LKAS. It provides an efficient procedure for designing a PWA output feedback controller, by means of a BMI optimization procedure, based on the search of a PWQL function.

According to (3), the partitions defined by the front wheel sideslip angles, $\alpha_{f}= \pm \bar{\alpha}_{f}$, depend on the state variables and control input. As mentioned in [14] the switching between the regions should depend only on the state $x$, so models in which there is a feed-through from $u$ to a nonlinearity should be avoided. This can be done by the inclusion of a first order 


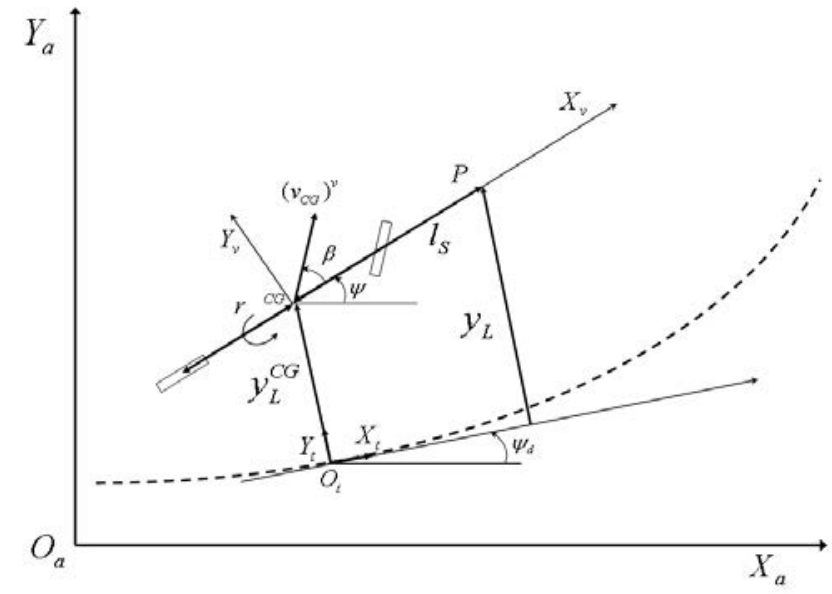

Fig. 2. Single track vehicle model.

actuator which is also useful to enforce continuity of the control input at the boundaries. Therefore, the steering angle is included as an additional state to the system as follows:

$$
\dot{\delta}_{f}=-\tau \delta_{f}+u_{c},
$$

where $u_{c}$ becomes the control input of the augmented plant and $\tau=10$.

As most vehicles have understeering behavior, it is assumed that the front tire forces tend to saturate firstly, therefore only the front tire forces are approximated by PWA functions according to (4), and a simply linear approximation is assumed for the rear tires. Nevertheless, an analogous approach can be considered in order to design assistances for oversteering vehicles.

Disturbances and exogenous inputs, such as the road curvature, are not taken into account in the control synthesis, therefore the PWA model describing the vehicle dynamics, positioning (10) and first order actuator (12) can be written as:

$$
\left\{\begin{array}{l}
\dot{x}=A_{i} x+B_{i} u+a_{i} \\
y=C_{i} x
\end{array}\right.
$$

with $x=\left[\beta, r, \psi_{L}, y_{L}, \delta_{f}\right]^{T}$ and $y$ is the system output. The matrices defining the dynamics are:

$$
\begin{gathered}
A_{i}=\left[\begin{array}{ccccc} 
& A_{i}^{p} & & B_{i}^{p} \\
0 & 0 & 0 & 0 & -\tau
\end{array}\right], \\
B_{i}=\left[\begin{array}{lllll}
0 & 0 & 0 & 0 & \tau
\end{array}\right]^{T}, a_{i}=\left[\begin{array}{lll}
\left(a_{i}^{p}\right)^{T} & 0
\end{array}\right]^{T},
\end{gathered}
$$

Since the vehicle sideslip angle is not available for measurement, the matrix defining the system output $y$ is given by:

$$
C_{i}=\left[\begin{array}{lllll}
0 & 1 & 0 & 0 & 0 \\
0 & 0 & 1 & 0 & 0 \\
0 & 0 & 0 & 1 & 0 \\
0 & 0 & 0 & 0 & 1
\end{array}\right] .
$$

Each of the regions $R_{i}$ (with $i=1,2,3$ ) can be described as the intersection of the half spaces:

$$
R_{i}=\left\{x \mid H_{i}^{T} x-g_{i}<0\right\}
$$

Since these regions are slab, it is preferred to express them as degenerated ellipsoids, as suggested in [13] and [14], because they describe exactly the slab regions and it often requires fewer parameters than the polytopic description (16).

The degenerated ellipsoids can be described by:

$$
\left\|E_{i} x+f_{i}\right\|_{2} \leq 1 \quad \forall x \in R_{i},
$$

and if $R_{i}=\left\{x \mid \underline{d}<c_{i}^{T} x<\bar{d}\right\}$ then the degenerated ellipsoid is described by $E_{i}=2 c_{i}^{T} /(\bar{d}-\underline{d})$ and $f_{i}=-(\bar{d}+\underline{d}) /(\bar{d}-\underline{d})$, as shown in [13].

For the PWA system (13), the ellipsoids describing the slab regions are:

$$
\begin{array}{ll}
E_{1}^{T}=\left[-1,-\frac{l_{f}}{v}, 0,0,1\right] \frac{2}{-\bar{\alpha}_{f}-\underline{d}}, & f_{1}=-\frac{-\bar{\alpha}_{f}+\underline{d}}{-\bar{\alpha}_{f}-\underline{d}}, \\
E_{2}^{T}=\left[-1,-\frac{l_{f}}{v}, 0,0,1\right] \frac{1}{\bar{\alpha}_{f}}, & f_{2}=-\frac{\bar{\alpha}_{f}-\bar{\alpha}_{f}}{\bar{\alpha}_{f}+\bar{\alpha}_{f}}=0, \\
E_{3}^{T}=\left[-1,-\frac{l_{f}}{v}, 0,0,1\right] \frac{2}{d-\bar{\alpha}_{f}}, & f_{3}=-\frac{\bar{d}+\bar{\alpha}_{f}}{d-\bar{\alpha}_{f}},
\end{array}
$$

where $\underline{d}$ and $\bar{d}$ can be arbitrarily set, such that $\underline{d}<-\bar{\alpha}_{f}$ and $\bar{d}>\bar{\alpha}_{f}$.

The ellipsoidal cell description is used to relax the constraints when searching the PWQL function. In order to ensure the continuity of the PWQL function across the boundaries, a parametric boundary description is needed [7].

Each facet boundary between the neighboring regions $R_{i}$ and $R_{j}$ is contained in the hyperplanes described by:

$$
\left\{x \mid c_{i j}^{T} x-d_{i j}=0\right\} .
$$

an the boundary parametric description can be obtained by:

$$
\bar{R}_{i} \cap \bar{R}_{j} \subseteq\left\{x\left|l_{i j}+F_{i j} s\right| s \in R^{n-1}\right\}
$$

where $F_{i j} \in R^{n \times n-1}$ (full rank) is the matrix whose columns span the null space of $c_{i j}$, and $l_{i j} \in R^{n}$ is given by $l_{i j}=c_{i j}\left(c_{i j}^{T} c_{i j}\right)^{-1} d_{i j}$

For the LKAS model (13) the parametric description of the boundaries is given by:

$$
\begin{aligned}
& F_{1,2}=\left[\begin{array}{cccc}
1 & 0 & 0 & 0 \\
0 & 1 & 0 & 0 \\
0 & 0 & 1 & 0 \\
0 & 0 & 0 & 1 \\
1 & \frac{l_{f}}{v} & 0 & 0
\end{array}\right], F_{2,3}=\left[\begin{array}{cccc}
1 & 0 & 0 & -\frac{l_{f}}{v} \\
0 & 0 & 0 & 1 \\
0 & 1 & 0 & 0 \\
0 & 0 & 1 & 0 \\
1 & 0 & 0 & 0
\end{array}\right], \\
& l_{1,2}=\left[\begin{array}{lllll}
\frac{\bar{\alpha}_{f}}{2+l_{f}^{2} / v^{2}}, & \frac{\bar{\alpha}_{f} l_{f} / v}{2+l_{f}^{2} / v^{2}}, & 0, & 0, & -\frac{\bar{\alpha}_{f}}{2+l_{f}^{2} / v^{2}}
\end{array}\right], \\
& l_{2,3}=\left[\begin{array}{lllll}
-\frac{\bar{\alpha}_{f}}{2+l_{f}^{2} / v^{2}}, & -\frac{\bar{\alpha}_{f} l_{f} / v}{2+l_{f}^{2} / v^{2}}, & 0, & 0, & \frac{\bar{\alpha}_{f}}{2+l_{f}^{2} / v^{2}}
\end{array}\right] \text {. }
\end{aligned}
$$




\section{A. PWA Output-feedback control synthesis}

Due to the symmetry of the lateral tire forces with respect to the origin, only regions $R_{1}$ and $R_{2}$ are considered in the control synthesis. The same gains obtained for region $R_{1}$ can be applied for region $R_{3}$.

The goal is to stabilize (13) with a PWA dynamic controller with state space representation of each region of the form:

$$
\left\{\begin{aligned}
\dot{x}_{c}(t) & =A_{c i} x_{c}+L_{i} y+b_{c i} \\
u & =K_{i} x_{c}+m_{i}
\end{aligned}\right.
$$

An augmented state $\tilde{x}=\left[x, x_{c}\right]^{T}$ is defined, so the closed loop system can be written as:

$$
\dot{\tilde{x}}=\tilde{A}_{i} \tilde{x}+\tilde{b}_{i}
$$

where;

$$
\tilde{A}_{i}=\left[\begin{array}{cc}
A_{i} & B_{i} K_{i} \\
L_{i} C_{i} & A_{c i}
\end{array}\right] \quad \text { and } \quad \tilde{b}_{i}=\left[\begin{array}{c}
b_{i}+B_{i} m_{i} \\
b_{c i}
\end{array}\right]
$$

The PWQL function for the augmented system (23) has the form:

$$
V_{i}(x)=\tilde{x}^{T} \tilde{P}_{i} \tilde{x}+2 \tilde{q}_{i}^{T} \tilde{x}+\tilde{r}_{i} .
$$

where $\tilde{P}_{i}=\tilde{P}_{i}^{T}, \tilde{P}_{i} \in R^{2 n \times 2 n}, \tilde{q}_{i} \in R^{2 n}$ and $\tilde{r}_{i} \in R$.

$V_{i}(x)$ is a Lyapunov function with a decay rate $\alpha_{i}$, for the region $R_{i}$ if, for a fixed $\epsilon \geq 0$,

$$
x \in R_{i},\left\{\begin{array}{l}
V_{i}(x)>\epsilon\left\|x-x_{e q}\right\|_{2} \\
\frac{d}{d t} V_{i}(x)<-\alpha_{i} V_{i}(x)
\end{array}\right.
$$

where $x_{e q}$ is the equilibrium point of the closed loop system.

Under strong lateral solicitations, the controller should bring the vehicle to the condition where the tire forces behavior is linear $\left(R_{2}\right)$. In order to do so, the desired equilibrium point of the closed loop system is placed at the origin $\left(x_{e q}=0\right)$. The equilibrium point must also be the extrema of the Lyapunov function candidate for the corresponding region (see [14]). In consequence, $q_{2}=0_{2 n \times 1}$ and $r_{2}=0$ to ensure that $V(0)=0$.

As the closed loop system has a single equilibrium point, the equilibrium points of $R_{1}$, denoted $x_{e q 1}$ and $R_{3}, x_{e q 3}$, must not be contained in its own region, enforcing a transition through the boundaries with $R_{2}$. This can be done using the constraint:

$$
\tilde{A}_{i} x_{e q_{i}}+\tilde{b}_{i}=0
$$

The stability of system (23) can be guaranteed by Sprocedure [6], using the ellipsoidal cell description (17) on conditions (26), if there exists $\tilde{P}_{i}=\tilde{P}_{i}^{T} \succ 0$, and positive constants $\lambda_{1}$ and $\gamma_{1}$ that satisfy:

$$
\left\{\begin{array}{cc}
{\left[\begin{array}{cc}
\tilde{P}_{1}-\epsilon I_{n}+\lambda_{1} \tilde{E}_{1}^{T} \tilde{E}_{1} & \tilde{q}_{1}+\epsilon x_{e q}+\lambda_{1} \tilde{E}_{1}^{T} f_{1} \\
* & \left(\begin{array}{c}
\tilde{r}_{1}-\epsilon x_{e q}^{T} x_{e q} \\
+\lambda_{1}\left(f_{1}^{T} f_{1}-1\right)
\end{array}\right)
\end{array}\right] \succ 0} \\
{\left[\tilde{P}_{2}-\epsilon I_{n}\right] \succ 0}
\end{array}\right.
$$

$$
\left\{\begin{array}{cc}
{\left[\begin{array}{cc}
\tilde{A}_{1}^{T} \tilde{P}_{1}+\tilde{P}_{1}+\tilde{A}_{1} & \tilde{P}_{1} \tilde{b}_{1}+\tilde{A}_{1}^{T} \tilde{q}_{1} \\
-\gamma_{1} \tilde{E}_{1}^{T} \tilde{E}_{1}+\alpha_{1} \tilde{P}_{1} & -\gamma_{1} \tilde{E}_{1}^{T} f_{1}+\alpha_{1} \tilde{q}_{1} \\
* & 2 \tilde{b}_{1}^{T} \tilde{q}_{1}+\alpha_{1} \tilde{r}_{1} \\
& -\gamma_{1}\left(f_{1}^{T} f_{1}-1\right)
\end{array}\right] \prec 0} \\
{\left[\tilde{A}_{2}^{T} \tilde{P}_{2}+\tilde{P}_{2}+\tilde{A}_{2}+\alpha_{2} \tilde{P}_{2}\right] \prec 0}
\end{array}\right.
$$

where $*$ means transposed and $\tilde{E}_{i}=\left[E_{i}, 0\right]$.

The parametric description of the boundaries (21) are adjusted to the augmented system as:

$$
\tilde{F}_{i j}=\left[\begin{array}{cc}
F_{i j} & 0 \\
0 & I
\end{array}\right] \text { and } \tilde{l}_{i j}=\left[\begin{array}{c}
l_{i j} \\
0
\end{array}\right]
$$

so that the continuity of the PWQL function candidate is ensured by:

$$
\left\{\begin{aligned}
\tilde{F}_{12}^{T}\left(\tilde{P}_{1}-\tilde{P}_{2}\right) \tilde{F}_{12} & =0 \\
\tilde{F}_{12}^{T}\left(\tilde{P}_{1}-\tilde{P}_{2}\right) \tilde{l}_{12}+\tilde{F}_{12}^{T} \tilde{q}_{1} & =0 \\
\tilde{l}_{12}^{T}\left(\tilde{P}_{1}-\tilde{P}_{2}\right) \tilde{l}_{12}+2 \tilde{q}_{1}^{T} \tilde{l}_{12}+\tilde{r}_{1} & =0
\end{aligned}\right.
$$

The switches of the controller can be driven only by the system outputs, therefore controller has to be designed with the structure of a regulator and an estimator. Thus, the front wheel sideslip angle must be computed with the estimate of the vehicle sideslip angle and the measurements of yaw rate and steering angle, according to (3). For that, the following constraints is added:

$$
\left\{\begin{array}{c}
A_{c i}=A_{i}+B_{i} K_{i}-L_{i} C_{i} \\
b_{c i}=B_{i} m_{i}+b_{i}+\left(A_{i}-L_{i} C_{i}\right) x_{e q}
\end{array}\right.
$$

Defining a performance criterion as: $J=\min \alpha_{i}$, The goal is to find $A_{c i}, K_{i} L_{i}, m_{i}, b_{c i}$, and a PWQL function (25) that maximize the performance criterion $J$. This optimization problem can be cast as a BMI as:

$$
\begin{aligned}
& \underset{i}{\max \min _{i} \alpha_{i}} \\
& \text { such that: (27), (28), (29), (31), (32), } \\
& \epsilon>0 \quad \gamma_{1}>0 \quad \lambda_{1}>0 \quad \alpha_{1,2}>l_{0}>0 \\
& -l_{1}<K_{i}<l_{1} \quad-l_{2}<m_{i}<l_{2}
\end{aligned}
$$

where $l_{0}$ is a scalar bound, $l_{1}$ and $l_{2}$ are vector bounds.

\section{B. Solution of BMI optimization problem}

The BMI problem (33) was solved using the V-K method, in which LMIs are solved in an iterative fashion. For that, the equilibrium point of the dynamics corresponding to the affine region $x_{e q 1}$, is chosen a-priori, such that it is not located in $R_{1}$. For the specific lane keeping problem, the algorithm becomes.

$V$-step: Given a fixed controller, and a fixed $\alpha_{i}$, solve:

Find: $P_{1}, q_{1}, r_{1}$ and $P_{2}$,

such that: (31), (28), (29), 
K-step: For $P_{1}, q_{1}, r_{1}$ and $P_{2}$ fixed at the previous step, solve:

$$
\begin{aligned}
& \underset{i}{\max \min _{i} \alpha_{i}} \\
& \text { such that (27), (28), (29), (32), } \\
& \epsilon>0, \quad \gamma_{1}>0, \quad \lambda_{1}>0, \quad \alpha_{1,2}>l_{0}>0, \\
& -l_{1}<K_{i}<l_{1}, \quad-l_{2}<m_{i}<l_{2},
\end{aligned}
$$

For each iteration of the K-step, the decay rates $\alpha_{1}$ and $\alpha_{2}$ must be greater than the value computed at the previous iteration. The loop must be repeated until there is no significant improvement on the cost or the LMIs become infeasible.

To design the initial controller for the first iteration on the $V$-step, it was supposed that all the state variables are available for measurement. A state feedback only for $R_{2}$ was computed using the algorithm from [9], and the gain for the observer was computed by pole-placement such that its poles are 10 times faster than the closed loop of the state feedback. The regulator and estimator structure was obtained from (32), and the same structure was considered for $R_{1}$ and $R_{3}$.

The resulting gains from the $\mathrm{V}-\mathrm{K}$ method after 4 iterations are:

$$
\begin{gathered}
K_{1}=[-0.8505-0.2780-1.5037-0.7683-0.5072] \\
K_{2}=[-0.7278-0.3558-1.7699-0.9394-0.8112], \\
K_{3}=K_{1}, \quad m_{1}=-m_{3}=0.023, \quad m_{2}=0 \\
\alpha_{1}=16.2349 \quad \text { and } \alpha_{2}=14.8648
\end{gathered}
$$

\section{Simulation Results}

Simulation have been carried out on the Matlab/Simulink environment, in order to verify the performance of the PWA controller. The dynamics of the model used for simulation consists of nonlinear 4-wheels vehicle according to [1], which is considered to capture the essential vehicle lateral steering dynamics:

$$
\begin{aligned}
m\left(\dot{v}_{y}+r v\right) & =f_{s f} \cos \delta_{f}+f_{s r} \\
J \dot{r} & =l_{f} f_{s f} \cos \delta_{f}-l_{r} f_{s r}
\end{aligned}
$$

where $v_{y}$ is the lateral velocity, and the lateral forces are given by (2). The sideslip angles $\alpha_{f, r}$ are given by:

$$
\begin{array}{r}
\alpha_{f(\text { left })}=\delta_{f}-\arctan \left(\begin{array}{c}
\frac{v \beta+r l_{f}}{v-\frac{a}{2} r} \\
\alpha_{f(\text { right })}=\delta_{f}-\arctan \left(\frac{v \beta+r l_{f}}{v+\frac{a}{2} r}\right.
\end{array}\right) \\
\alpha_{r(\text { left })}=-\arctan \left(\begin{array}{c}
\frac{v \beta-r l_{r}}{v-\frac{a}{2} r} \\
\alpha_{r(\text { right })}=-\arctan \left(\frac{v \beta-r l_{r}}{v+\frac{a}{2} r}\right)
\end{array}\right)
\end{array}
$$

The vehicle model has been submitted to the obstacle avoidance maneuver defined in ISO 3888-2 standard, at a constant longitudinal velocity of $v=21 \mathrm{~m} / \mathrm{s}$. Fig. 3 shows the displacement of the vehicle within the area delimited by the cones, confirming that the maneuver was successfully achieved.

It can be seem from Fig. 4 that the vehicle reaches the nonlinear behavior of tire lateral forces, overshooting the

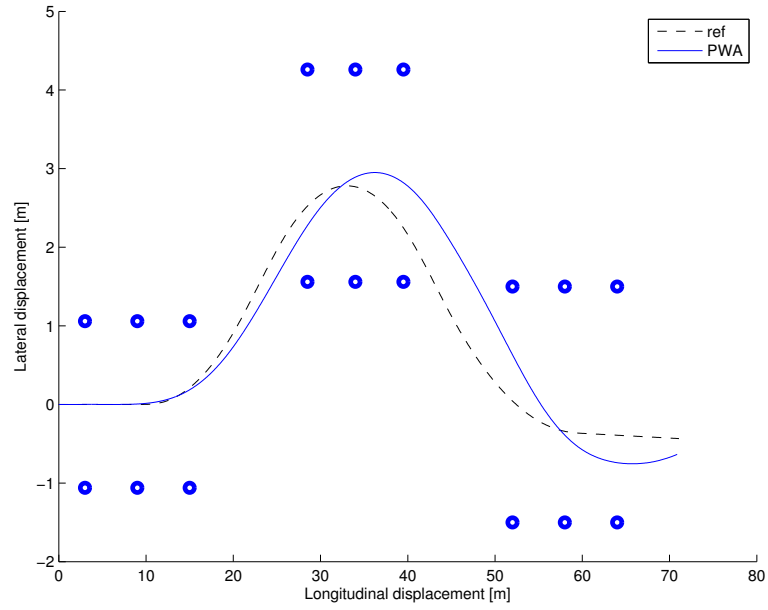

Fig. 3. Displacement of the vehicle equipped with PWA output-feedback controller in standardized maneuver ISO-3888-2.
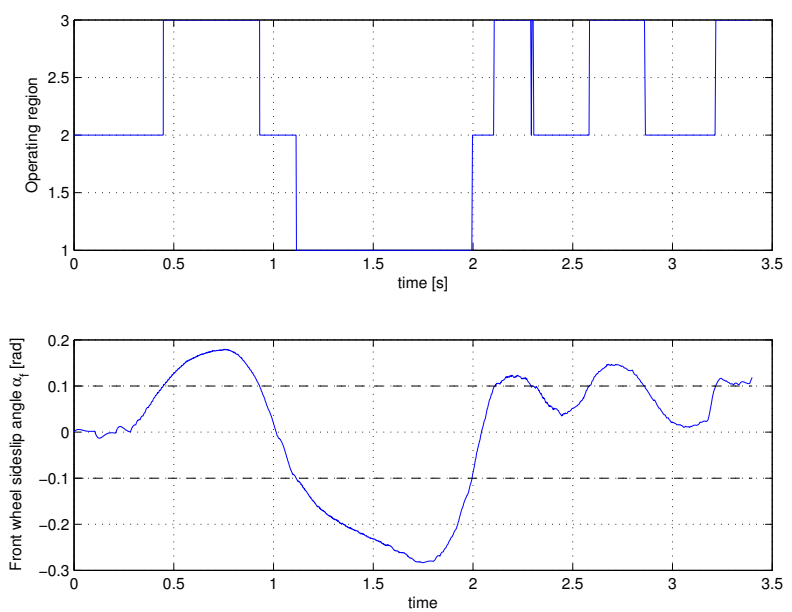

Fig. 4. Active region for PWA control and front wheels estimated sideslip angle

limit $\bar{\alpha}_{f}$. The corresponding operating regions from the switches of the controller are shown in the upper subplot Fig. 4, while the estimated front sideslip angle is shown in the bottom subplot.

The control input required to perform the maneuver, as well as the corresponding steering angle are depicted respectively in the upper and bottom subplots of Fig. 5 .

The vehicle sideslip angle and yaw rate are shown in subplots of Fig. 6. The relative yaw angle and lateral offset at look-ahead distance, representing the vehicle positioning on the road are depicted in Fig 7.

\section{CONCLUSION}

In this paper the design and simulation of a PWA controller for LKAS has been described. The nonlinear behavior of the lateral tire forces are approximated by PWA functions. As only understeering vehicles are considered in this work, the regions describing the PWA vehicle model can be exactly described as degenerated ellipsoids which are used to relax the conditions for the existence of a PWQL function. The 

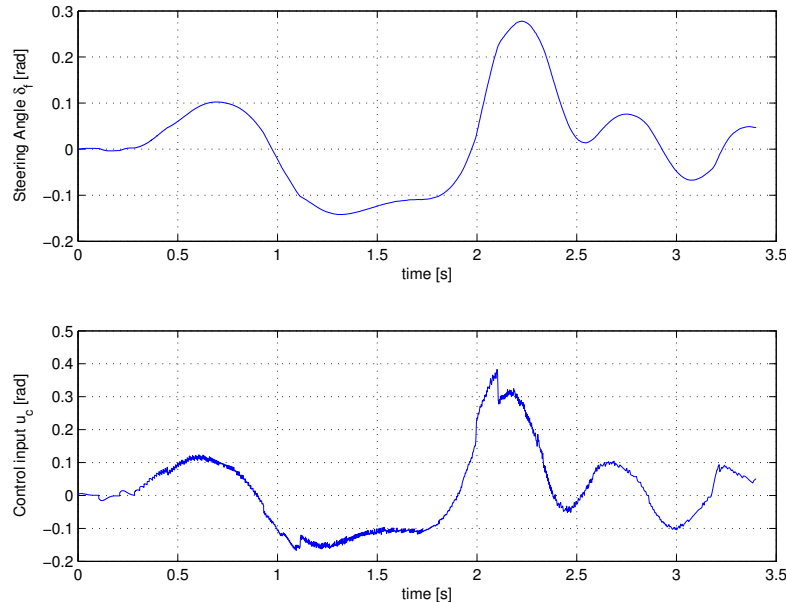

Fig. 5. Control input and Steering angle for vehicles with PWA controller
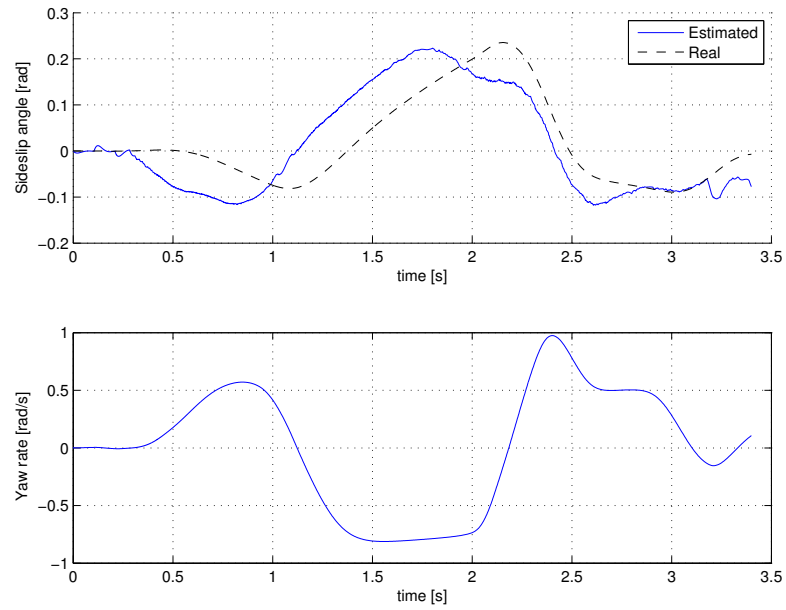

Fig. 6. Vehicle dynamics response
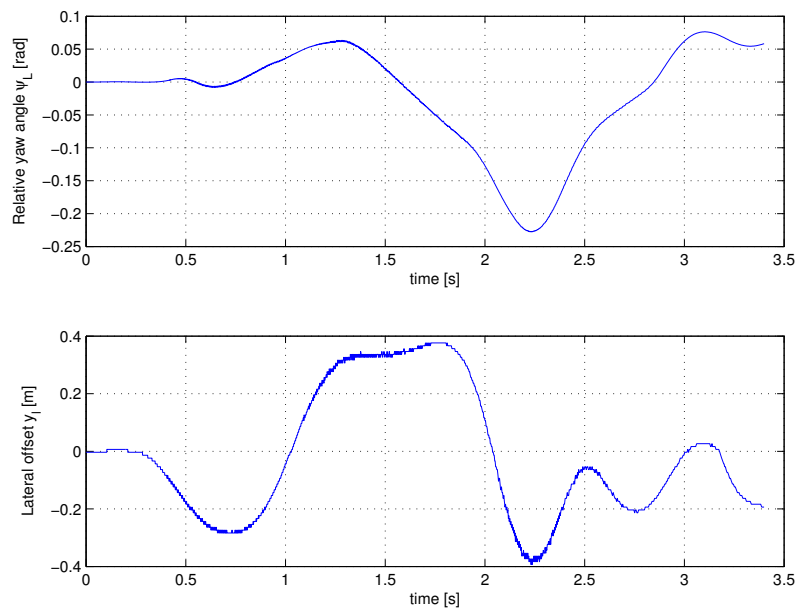

Fig. 7. Positioning state variables simultaneous search of a PWQL function and a PWA observer based output-feedback gain has been casted as a BMI optimization problem and its solution has been computed using the $\mathrm{V}-\mathrm{K}$ method in which the BMIs are transformed into two LMI optimization procedures that have been solved iteratively. The resulting PWA controller has been tested according to the double lane change standard maneuver ISO3888-2, showing that the PWA controller can handle the nonlinearity of the tyre lateral force. The switches are based on the state estimation, avoiding measurement of the vehicle sideslip angle, which requires a sensor that is not available in currently commercialized vehicles and are also very costly.

System robustness with respect to parameter variations, as for example vehicle longitudinal speed and load transfers should be taken into account in the future work as well as implementation on prototype vehicle.

\section{REFERENCES}

[1] J. Ackermann. Robust Control. Springer, London, 2002.

[2] A. Benine-Neto and S. Mammar. Piecewise affine state feedback controller for lane departure avoidance. In Intelligent Vehicles Symposium (IV), 2011 IEEE, pages 839 -844, june 2011.

[3] A. Benine-Neto, S. Scalzi, M. Netto, S. Mammar, and W. PasillasLepine. Vehicle yaw rate control based on piecewise affine regions. In Proceedings of the IEEE Intelligent Vehicles Symposium, San Diego, USA, pages 20-25, June 2010.

[4] D. Bernardini, S. D. Cairano, A. Bemporad, and H. Tseng. Driveby-wire vehicle stabilization and yaw regulation: A hybrid model predictive control design. In Joint 48th IEEE Conference on Decision and Control and 28th Chinese Control Conference, Shanghai, P.R. China, pages 7621-7626, December 2009.

[5] F. Borrelli, A. Bemporad, M. Fodor, and D. Hrovat. A Hybrid Approach to Traction Control. Springer Berlin / Heidelberg pp. 162174, 2001.

[6] S. Boyd, L. E. Ghaoui, E. Feron, and V. Balakrishnan. Linear Matrix Inequalties in System and Control Theory, chapter 7. SIAM, 1994.

[7] A. Hassibi and S. Boyd. Quadratic stabilization and control of piecewise-linear systems. In Proceedings of the IEEE American Control Conference, Philadelphia, PA, pages 3659-3664, 1998.

[8] S. Mammar, N. Minoiu-Enache, S. Glaser, B. Lusetti, and A. BenineNeto. Lane keeping automation at tire saturation. In Proceedings of the IEEE American Control Conference, Baltimore, pages 6466-6471, June 2010.

[9] N. Minoiu-Enache, B. Lusetti, S. Mammar, and M. Netto. Assistance active de prévention des sorties de voie. prise en compte des virages. Journal Européen des Systèmes Automatisés, 43(6):615-646, 2009.

[10] E. Ono, S. Hosoe, H. D. Tuan, and S. Doi. Bifurcation in vehicle dynamics and robust front wheel steering control. IEEE Trans. on Control System Technology, 6(3):9-14, May 1998.

[11] H. Pacejka. Tire and Vehicle Dynamics. Elsevier ButterworthHeinemann, 2004.

[12] G. Palmieri, M.Baric, and F. Borrelli. Constrained robust design of lateral vehicle dynamics control systems. In Proceeding of AVEC, aug 2010.

[13] L. Rodrigues and S. Boyd. Piecewise-affine state feedback for piecewise-affine slab systems using convex optimization. Systems and Control Letters, 54:835-853, 2005.

[14] L. Rodrigues and J. P. How. Observer based control of piecewise-affine systems. Int. J. Control, 76(5):459-477, 2003.

[15] E. J. Rosseter and J. C. Gerdes. Lyapunov based performance guarantees for potencial field lane keeping assistance system. Journal of Dynamic Systems, Measurement and Control - Transactions of the ASME, 128:510-522, September 2006.

[16] S. E. Shladover. Path at 20 - history and major milestones. IEEE Transactions on Intelligent Transportation Systems, 8(4):584-592, December 2007. 